\title{
La gota serena, el glaucoma antes del siglo XX
}

\section{Glaucoma before the $20^{\text {th }}$ century}

Jaime Lozano-Alcázar*

Fundación Hospital Nuestra Señora de la Luz, Ciudad de México, México

\section{Resumen}

En tiempos remotos, la ceguera se explicaba, a menudo, por un cuadro llamado la gota serena u otros términos como ambliopía, amaurosis, glaucoma o sufussio, como se llamaba también a la catarata. Los cuadros de gota serena, que hoy llamaríamos glaucoma, podían deberse a la exposición al sol, al aire, a ciertos alimentos y, particularmente, a los excesos sexuales, en especial la ophthalmía onanística, argumento como causa de ceguera para amedrentar a los jóvenes «viciosos». Es interesante dar una mirada a la evolución que a lo largo de los siglos va teniendo el conocimiento del glaucoma, o mejor dicho, los glaucomas.

\section{Palabras clave: Glaucoma. Gota serena. Historia}

\begin{abstract}
In ancient times, blindness was often explained by a clinical appearance of gutta serena or other terms such as amblyopia, amaurosis, glaucoma or sufussio, a term used for cataracts. Gutta serena, which today we call glaucoma, could be due to sun or air exposure, certain foods and particularly sexual excesses, especially onanistic ophthalmia, an argument as a cause of blindness to intimidate "vicious" young people. It is interesting to take a look at the evolution of knowledge about glaucoma over the centuries.
\end{abstract}

Key words: Glaucoma. Gutta serena. History.

El término glaucoma aparece desde los aforismos de Hipócrates, pero no designa una entidad clínica determinada, sino solamente aquellos casos de ceguera en edad avanzada en los que la pupila tomaba una apariencia glaseada: «si la pupila toma una coloración parecida a la del mar, la visión es destruida y a menudo le sigue la del otro ojo". El color al que se hace referencia es, sin duda, el verde 0 , mejor, una coloración verdosa, de ahí la etimología del término. Evidentemente, se aplicaba de manera genérica, sin alguna connotación patológica específica, simplemente patologías que coincidían en causar ceguera y que la pupila tomara una coloración glaseada de tono verdoso; seguramente incluía el glaucoma absoluto, entre otras. A las entidades clínicas relacionadas también se les aplicaban indistintamente términos como ambliopía, amaurosis o, más comúnmente, gutta serena. Con frecuencia se ubicaba en el cristalino la génesis de la ceguera, pues era 
considerado entonces el órgano esencial de la visión, que se alteraba debido a los "espíritus visuales». Para algunos autores, este mal se debía a que los espíritus no podían transitar libremente por el nervio óptico hasta el cerebro.

A principios de la era cristiana, Celso (25 a. C.-50 d. C.), Rufo de Éfeso (95-117 d. C.) y Galeno (131-210 d. C.) consideraban que la ceguera podía ser causada por dos entidades o conjunto de enfermedades: la suffussio, asociada a un humor maligno enfrente del cristalino, en la que el paciente podía percibir la luz, y era susceptible de remediarse mediante cirugía, aun cuando la pupila se viese blanca, es decir, la catarata; y la otra debida a desecación del cristalino, en la que el paciente llegaba a perder totalmente la visión, la pupila tomaba un aspecto glaseado de coloración verde-azulosa y la pérdida era irrecuperable, es decir, el glaucoma.

Juan Naval, médico de los reyes de España, en 1796, en su libro De la ophthalmía y sus especies explica que se le llamó gota serena "porque en otros tiempos se creyó se debía a un derrame de la linfa, y se añade serena porque el ojo no está nada turbio, como en la catarata; si se cierra el ojo sano y se expone a la luz el ciego, la pupila de este, lejos de contraerse, algunas veces se dilata... y es señal de la gota serena ... también se le llama suffusio nigra». Refiere que el famoso oculista francés Mr. De Saint-Ives, divide la gota serena en perfecta e imperfecta. En esta última, el ojo ve aún «alguna cosa». El médico español, continúa: «fácilmente se conocerá el grado de esta enfermedad, haciendo que el paciente mire a un libro cerrando el ojo sano, de este modo no verá más que una cierta porción de la página, al paso que con el ojo sano la verá toda». ¡El deterioro del campo visual por glaucoma!

Con el paso del tiempo, el término gutta serena, castellanizado como gota serena, se fue asociando con lo que hoy conocemos como glaucoma. En 1817, el Diccionario de la Real Academia Española de la Lengua define a la gota serena como la privación total de la vista sin señal exterior ni visible en los ojos (glaucoma). Hacia mediados del siglo XIX, antes del oftalmoscopio, ya era común enfatizar que no se debía confundir la catarata con el glaucoma, enfermedad en la que «el médico y el enfermo nada vemos» (el médico no apreciaba alteraciones en el ojo).

Las causas de la gota serena podían ser la exposición al sol, al aire, ciertos alimentos, etc., la congestión de sangre en la cabeza y, particularmente, los excesos sexuales, en especial la ophthalmía onanística, argumento muy utilizado como causa de ceguera para amedrentar a los jóvenes «viciosos»"-7.

En 1709, Michael Brisseau, después de examinar al Dr. Pierre Bourdelot, el médico ciego de Luis XIV, asoció la catarata con la opacificación del cristalino. Así mismo, dedujo que el glaucoma o gota serena no se debía a una anomalía del cristalino. Sin embargo, años después, en 1748, Boerhaave (probablemente el famoso médico Herman Boerhaave, llamado el Hipócrates holandés, que ya había muerto en el año señalado por la fuente) argumentaba que el glaucoma era un tipo de catarata que empezaba con dolor y terminaba con ceguera. Ninguno de los dos menciona algún cambio en la consistencia del globo ocular ${ }^{1}$.

En el siglo X, el médico árabe Al-Tabari en su Libro del tratamiento hipocrático escribió de un proceso inflamatorio crónico del ojo que cursaba con aumento del tono del globo. Pocos siglos después, Samas-al-Din (?-1348), de El Cairo, reseñó 153 enfermedades del ojo y sus anexos; describe una «migraña del ojo» 0 «cefalea de la pupila», caracterizada por dolor ocular, cefalea en hemicráneo, endurecimiento de los humores del ojo, seguido de dilatación de la pupila, catarata y ceguera ${ }^{1}$.

El cirujano itinerante inglés Sir Richard Bannister, Fellow of the Royal College of Surgeons, autor del primer libro de Oftalmología en inglés, con enseñanzas originales, en 1622 hizo el primer reconocimiento claro de la asociación del aumento del tono ocular y el glaucoma, pues distingue entre la catarata curable o gutta obscura y la gutta serena en la que "... the humor settled in the hollow nerves, be growne to any solid or hard substance, it is no posible to be cured..."* (el humor alojado en los espacios de los nervios madura (sic) a una sustancia sólida o dura, no es posible curarla), continúa "... If one feele the Eye by rubbing upon the Eie-lids, that the Eye be growne more solid and hard tan naturally it should be..." (Si uno siente al ojo palpando a través de los párpados el ojo habrá madurado (sic) hacia más sólido y duro de lo que naturalmente debería ser). Agregó que el curso de la enfermedad era largo, la pupila se volvía fija y se perdía la percepción de luz'.

En el siglo XVIII se asoció frecuentemente el glaucoma a la inflamación. Si la pupila del ojo inflamado tomaba una coloración azul-verdosa, el pronóstico visual era malo, sin alusión al tono ocular. Sin embargo, Johann Zacharias Platner, en 1738, distinguía dos tipos

* Se conserva la ortografía de la época. 
de enfermedad, una debida a inflamación del cristalino, en la que el ojo devenía duro, y otra a inflamación del vítreo, en la que el ojo se hacía blando.

En el siglo XVIII, en España, adquirió fama el monje benedictino Benito Jerónimo Feijoo (1676-1764), que ejerció la Cátedra de Teología en la Universidad de Oviedo y cuyos intereses abarcaron también diversos aspectos humanos y mundanos. Ya en su madurez, de 1726 a 1740, publicó en nueve volúmenes, bajo el título de Teatro crítico universal, sobre «todo género de materias, para desengaño de errores comunes»; en los tomos II y III se refiere a aspectos oculares; además de hacer gala de varios conocimientos acertados en cuanto a anatomía y fisiología del aparato de la visión -aunque desde luego hay otros conceptos erróneos-, relata: «una conmoción, o impresión, que hacen los rayos visuales en la retina, se propaga en un momento por el nervio óptico, que es continuación de ella, hasta el origen del nervio, que está dentro del cerebro ... En llegando la impresión al origen del nervio óptico, resulta, o se excita en el alma aquella percepción del objeto, que llamamos Visión". Una de las enfermedades que reseña el autor es la gota serena, que con toda posibilidad corresponde al glaucoma: «el órgano particular de la vista está perfectamente bien dispuesto; sin embargo, el sujeto, que padece esta enfermedad, nada ve, no por otra razón sino porque en virtud de la indisposición de los nervios ópticos no se propaga hasta el cerebro la impresión, que los objetos hacen en el ojo ... comúnmente los médicos explican este defecto ... por la falta de fluencia de los espíritus animales del cerebro a los ojos, cuyo curso impide la obstrucción, o compresión del nervio óptico». Mediante la gota serena, Feijoo pretende explicar la función visual del alma: «Si los ojos fuesen el órgano propio de la potencia visiva, entretanto que ellos estuviesen sanos, vivos y animados, no podría faltar la vista; pero esto es falso: luego, aquella enfermedad que llamamos gota serena, y que proviene únicamente de la obstrucción del nervio óptico, siendo perfecta la obstrucción, falta enteramente la vista; con todo, los ojos están vivos y animados» ${ }^{8}$.

En la Real Academia de Cirugía de Paris, Arrachat, en 1786, describió el cuadro agudo del glaucoma en el que el ojo se vuelve doloroso e inflamado, el iris pierde su color, la pupila se dilata y toma una coloración verdosa y evoluciona a la ceguera. Josef de Beer, de Viena, en 1792 describe un cuadro de «inflamación del iris» con los signos distintivos del glaucoma, sin mencionar hipertensión y que asocia a una «diátesis gotosa».

En el siglo XIX se correlacionan definitivamente hipertensión ocular y glaucoma. La primera descripción magistral aparece en el Traite des maladies de yeux, de Antoine-Pierre Demours, en 1818, libro en el que el autor dejó a la posteridad el acervo de conocimientos médicos de su padre, el Dr. Pierre Demours. Describió: «le globe devient dur au toucher»; describió por primera vez los clásicos halos de colores (l'arc-en-ciel) alrededor de las luces. En 1823, G. J. Guthrie, en Londres, reconoció una entidad llamada glaucoma en la que «si el ojo es palpado se encontrará más duro que lo natural». El glaucoma se seguía haciendo depender de una condición inflamatoria, algunos autores contemporáneos describían cuadros de un síndrome inflamatorio agudo que afectaba el vítreo y la coroides, llamado oftalmía artrítica, y otra forma crónica de la misma condición cuyo cuadro corresponde al hoy llamado glaucoma absoluto. Franciscus $\mathrm{C}$. Donders consideraba que la forma no congestiva (simple) era un miembro de la misma familia. Ya no hubo duda de la asociación definitiva de hipertensión ocular con el glaucoma crónico o agudo a partir de los postulados de William Mackensie (1791-1868) en 1835, y distinguió entre glaucoma crónico y glaucoma agudo ${ }^{1,9}$.

Otro hito en el devenir histórico del conocimiento del glaucoma estuvo dado por el oftalmoscopio de Helmhotz difundido por Albrecht von Graefe a mediados del siglo XIX, cuando se empezaron a difundir las modificaciones en la excavación papilar y proliferaron los dibujos describiendo las alteraciones. Von Graefe lo clasificó en tres categorías, aún vigentes: agudo, crónico y secundario. Otro avance no menor, en las primeras décadas del siglo XX, fue el examen clínico del ángulo de la cámara anterior, en el que destaca nuestro compatriota el Dr. Manuel Uribe y Troncoso, y el conocimiento de la fluídica del humor acuoso'.

Louis de Wecker (1832-1906), destacado oftalmólogo francés, nacido en Frankfurt am Main, en su Manuel d'phthalmologie. Guide practique(1889), señala que el ojo se vuelve glaucomatoso en el momento en que el equilibrio entre la secreción y la excreción del órgano se rompe a favor de la cantidad de líquido que contiene fisiológicamente el globo; esta ruptura de equilibrio provocará un aumento de la presión intraocular, una acentuación de tensión y una distención consecutiva de las partes menos resistentes del globo ocular. Distingue cuatro tipos de glaucoma: 1) prodrómico, 2) crónico simple, 3) crónico irritativo, 4) irritativo agudo y fulminante. En el glaucoma crónico simple describe el aumento de la excavación papilar y la pérdida del campo visual periférico. Añade: para el prodrómico son generalmente sujetos de temperamento nervioso e irritable. Se les debe recomendar vida al aire libre, paseos a pie por el campo, baños de mar, una gota de 
pilocarpina al dormirse o durante el día aplicaciones periódicas de eserina; si el problema continúa, habrá que recurrir a una esclerotomía conjunta con masaje periódico y pilocarpina; y si los ataques prodrómicos se intensifican, habrá que recurrir a la iridectomía ${ }^{10}$.

El tratamiento general que preconiza Wecker era el que se recomendaba antes del siglo XX; medidas higiénicas y dietéticas, complementadas con los acostumbrados métodos antiflogísticos, como purgas, vomitivos, ventosas, sangrías secas (con sanguijuelas) y húmedas mediante flebotomías y medicamentos «que aumenten los espíritus y que en parte atenúen y en parte revelen, avoquen y disipen la viscosidad de los humores permitiendo (pero también interpolando) los purgantes idóneos y los eméticos bien indicados». "De provenir la gota serena de mal venéreo ... se necesita el mercurio»2-6.

Se cree que, entre los aztecas, el glaucoma se trataba con una planta, la Ohuaxocoyolin, mezclada con excremento, que se aplicaba a los ojos.

A fines del XVIII, el Dr. H Collin, de Viena, popularizó el manejo de la gota serena con las gotas de árnica (Linneo: Arnica Montana o Fherio panacea)2.

Poco después que se descubriera la electricidad, esta se puso de moda con fines terapéuticos. En España, en 1799, el Dr. Pedro Aguilar, en vista de que los tratamientos convencionales no surtían efecto en un paciente aquejado de gota serena, «se pasó a la electricidad» mediante «la electrificación por baño» con un disco de cristal que se aplicaba a la órbita del paciente, en sesiones de media hora que, luego de varios días, hubieron de suspenderse por cefalea intensa, pero tras un periodo de tratamiento con sanguijuelas se reiniciaron las sesiones con electricidad, días después, el paciente «salió de este hospital curado a satisfacción de D. Pedro de Aguilar» ${ }^{11}$.

En el año 1836, en el Periódico de la Academia de Medicina de Mégico (sic) (este organismo fue el primero en tomar el nombre de academia, su existencia fue de pocos años, la actual Academia Nacional de Medicina de México es la tercera con el nombre), se reseña que los tratamientos antiguos de la gota serena consistían en causar irritación a la superficie de la córnea, empleaban, por ejemplo, fricciones con las barbas de una espiga de trigo; un Dr. Taylor frotaba la córnea con piedra pómez o con una lámina de oro muy áspera; pero el método que se preconiza en el artículo es el del Dr. Serre D'uzes, «más sencillo, más fácil y menos doloroso", consiste en el uso de nitrato de plata en lápiz, que con gentileza se aplica en el borde inferior de la córnea transparente "hasta que aparezca una nubecita en el punto cauterizado». Luego, se lava el ojo con abundante agua para disolver el excedente del cáustico «y calmar la irritación pasajera que resulta de su aplicación». El Dr. Serre refiere ocho casos tratados con su método, la mayoría por «amaurosis que se había presentado casi de repente y cuya duración tenía fecha muy nueva». El autor concluye "es un remedio precioso en muchas circunstancias en que el arte hasta ahora era casi impotente ${ }^{12}$.

Naturalmente, cuando empezó a utilizarse el oftalmoscopio inventado por Hermann von Hemlholz se disparó el conocimiento del glaucoma y la literatura al respecto, así como de todas las afecciones del fondo ocular. Una contribución no menor al conocimiento del glaucoma surgió pocos años después, fue el diseño y uso de tonómetros oculares. Los primeros diseños fueron de indentación, en 1863, von Graefe, Donders y Hamer presentaron sus inventos, luego Dor, en 1865, Priestley Smith, en 1879, Koster, en 1895. Todos estos modelos fueron desplazados por el tonómetro de Hjalmar August Schiotz (1850-1927), producido en 1905. Los primeros tonómetros de aplanación fueron diseñados por Weber en 1867 y Maklakow en 1895. Este último fue el que prevaleció. Ya en el siglo XX aparecerían otros muchos tonómetros de aplanación. Las alteraciones del campo visual se conocían desde la antigüedad, sin embargo, la contribución de Janik Peterson Bjerrum (1851-1920) y su pantalla tangencial, a partir de 1889, fue decisiva para el estudio del glaucoma, complementada por su discípulo Henning Roenne ${ }^{13}$.

Existen antecedentes de cirugías para el glaucoma anteriores a la descripción y difusión que hizo Albrecht von Graefe (1828-1879) de la iridectomía en 1856 o $1857^{1,10,14}$, que fue rápidamente adoptada en todo el mundo; aunque él mismo aceptaba que la idea de hacer una pequeña excisión en el iris había sido postulada previamente por Louis Auguste Desmarres. En efecto, desde 1728, William Cheselden ejecutaba la iridotomía al puncionar el iris con una aguja, otros autores usaron posteriormente diversos tipos de lancetas, y penetraban por la córnea en lugar de por la esclerótica. En 1872, durante un congreso en Londres, William Bowman hizo una comunicación sobre la formación de la pupila por incisión, la llamada iridotomía óptica. La persona a la que se practicaba una iridotomía o una iridectomía se les decía que era una pupila artificial antiflogística, que prevenía las consecuencias de la seclusión u oclusión pupilar en los casos de «iridocoroiditis supurativa" que se presentaba en uno de cada 500 o 600 casos de cirugía intraocular, según afirmación de Carl Ferdinand von Arlt (1812-1887) ${ }^{15}$. La iridectomía óptica fue muy popular para el manejo de cataratas 
congénitas nucleares, se practicaba en los cuadrantes mediales inferiores. Al principio de mi práctica me tocó ver adultos mayores a quienes se había practicado esa intervención cuando eran niños. Una curiosa intervención quirúrgica para el glaucoma fue propuesta por Hanccock, la sección del músculo ciliar, introducía una lanceta de catarata en la parte inferior y externa del borde de la córnea en su unión con la esclerótica, la punta del cuchillete era pasada de adelante a atrás y de arriba abajo para que las fibras de la esclerótica fueran divididas oblicuamente una extensión de un octavo de pulgada. Afirma que la operación rara vez es seguida de inflamación o síntomas molestos ${ }^{16}$.

El tratamiento medicamentoso fue más tardío. En 1876, Adolf Weber (1829-1915), discípulo y amigo de von Graefe, implantó la aplicación de pilocarpina. Prácticamente al mismo tiempo, Ludwig Laqueur (1839-1909), en 1876-1877, impulsó la aplicación tópica de eserina ${ }^{1,10,14}$.

Los conceptos etiopatogénicos y terapéuticos del pasado nos parecen hoy absurdos, ridículos ... ¿Qué pensarán de nuestros conocimientos actuales las generaciones futuras?

\section{Bibliografía}

1. Duke-Elder S, Barrie J. Section III: Glaucoma and Hypotony. In: Duke-Elder S, ed. System of Ophthalmology, Vol. XI: Diseases of the Lens and
Viterous; Glaucoma and Hypotony. 1969. London, Kimpton. 1995. p: $379-88$

2. López de Letona C. Un curioso remedio para la curación de la amaurosis (siglo XVIII), Arch Soc Esp Oftalmol. 2004;79 (1):41-2.

3. López-Letona C. El tratado elemental de las enfermedades de los ojos de Lorenzo Baró (1889). (II). Arch Soc Esp Oftalmol. 2005;80(6):369-70.

4. Heister L. De las principales enfermedades de los sentidos. En: Compendio de toda la medicina práctica. Madrid: Imprenta de los sentidos; 1776. p. 83-4, 92-3.

5. Medicina Doméstica o Tratado Completo, Por Guillermo Buchan, M. D. del Real Colegio Médico de Edimburgo, Traducido por Pedro Sinnot, Presbítero. Segunda Edición, Tomo III. Madrid, de la Imprenta Real, MDCCXCII. P:325, 326, 327, 328, 329. Citado por Sampedro A, Arch Soc Esp Oftalmol. On line.

6. Naval Juan: De la Ophthalmía y sus Especies. Parte Segunda. Madrid. En la Imprenta Real,1796.https://books.google.es/books?id=3kZ07MTTgV0C\&pg=PR6\&focus=viewport\&dq=gota+serena $\& \mid r=\& h l=e s \& o u t p u t=h t-$ ml_text

7. Suárez E, Junceda J, Junceda C. Visión y sexo. Arch Soc Esp Oftalmol. 2001(8):515-6.

8. Sampedro A, Barbón-García JJ. Cuestiones oftalmológicas en la Obra de Feijoo (i). Arch Soc Esp 2008. 83(1):63-6.

9. Balanik G. Study of Glaucoma has a Long Hystoryhttp://www.escrs.org/ publications/eurotimes/07aug/studyofglaucoma.pdf

10. De Wecker L, Masselon J. Manuel D'Ophthalmologie. Guide Practique. Paris: Lecrosnier et Babé, Libraires-Editeurs; 1889. p: 402-50.

11. López de Letona C. Cirujanos oculistas en la Gaceta de Madrid (S.XVIII) (I); Arch Soc Esp Oftalmol. 2007; 82(7):459-60.

12. Periódico de la Academia de Medicina de Mégico (sic) o. Tomo I. Julio 15 de 1836. Mégico: Imprenta de Galván; 1836. p. 30-1.

13. Duke-Elder S, Smith RJH. Section III Clinical Methods of Examination, Hypotony. En: Duke-Elder S. ed. System of Ophthalmology. Vol. XVII: The Foundations of Ophthalmology. London: Kimpton; 1995. p. 339, 349, 394.

14. Duke-Elder S, Barrie J. Section III: Glaucoma and Hypotony. En: Duke-Elder S, ed. System of Ophthalmology. Vol. XI: Diseases of the Lens and Viterous; Glaucoma and Hypotony. London: Kimpton; 1995. p. 503.

15. De Wecker L, Masselon J. Crirurgie Oculaire. Paris: Octave Dion, Editeur; 1879. p. $115,137,142$.

16. Meyer E. Traité Pratique des Maladies des Yeux. Paris: Hlauwereyns, Librarie-Éditeur; 1873. p. 275. 